\title{
Flow-metabolism dissociation in the pathogenesis of levodopa-induced dyskinesia
}

\author{
Vincent A. Jourdain, Chris C. Tang, Florian Holtbernd, Christian Dresel, Yoon Young Choi, Yilong Ma,
} Vijay Dhawan, and David Eidelberg

Center for Neurosciences, The Feinstein Institute for Medical Research, Manhasset, New York, USA.

Levodopa-induced dyskinesia (LID) is the most common, disruptive complication of Parkinson's disease (PD) pharmacotherapy, yet despite decades of research, the changes in regional brain function underlying LID remain largely unknown. We previously found that the cerebral vasomotor and metabolic responses to levodopa are dissociated in PD subjects. Nonetheless, it is unclear whether levodopa-mediated dissociation is exaggerated in LID or distinguishes LID from non-LID subjects. To explore this possibility, we used dual-tracer positron emission tomography to quantify regional cerebral blood flow and metabolic activity in 28 PD subjects (14 LID, 14 non-LID), scanned before and during intravenous levodopa infusion. Levodopa-mediated dissociation was most prominent in the posterior putamen $(P<0.0001)$ and greater in LID than in non-LID and test-retest subjects. Strikingly, LID subjects also showed increased sensorimotor cortex (SMC) activity in the baseline, unmedicated state. Imaging data from an independent PD sample (106 subjects) linked these differences to loss of mesocortical dopamine terminals in advanced patients. In aggregate, the data suggest that LID results from an overactive vasomotor response to levodopa in the putamen on a background of disease-related increases in SMC activity. LID may thus be amenable to treatment that modulates the function of these 2 regions.
Conflict of interest: The authors have declared that no conflict of interest exists.

Submitted: January 18, 2016 Accepted: August 18, 2016 Published: September 22, 2016

Reference information: JCI Insight. 2016;1(15):e86615. doi:10.1172/ji.insight.86615.

\section{Introduction}

Although levodopa remains the gold standard pharmacological treatment of Parkinson's disease (PD) $(1,2)$, long-term administration eventually produces levodopa-induced dyskinesias (LID). LID appears within the first 5 years of drug treatment in approximately $40 \%$ of PD patients, and nearly all PD patients are affected after 10 years (3). The mechanisms underlying PD-related dyskinesias are multifactorial, with studies in various experimental models providing evidence that pre- and postsynaptic changes in striatal dopaminergic terminals, downstream changes in gene expression, and alterations in non-dopaminergic transmitter systems are all involved (4). The changes in brain function that mediate LID at the systems level, however, remain poorly understood.

In human subjects with $\mathrm{PD}$, positron emission tomography (PET) studies have revealed that the pharmacokinetics of levodopa change over the course of the disease (5). As PD progresses, levodopa induces a faster, larger, but less sustained dopamine release in the putamen, which is linked to response fluctuations and, ultimately, LID (5). Yet this pulsatile pattern of stimulation of the dopamine receptors occurs in PD patients without LID as well as those with LID, indicating that other mechanisms must be involved. One contributing factor could be the integrity of striatal serotonergic terminals. Serotonergic neurons can convert levodopa into dopamine, and store and release it as a "false" neurotransmitter, but as they possess neither dopamine transporters (DATs) nor dopamine autoreceptors, their release of dopamine is unregulated. If the serotonergic terminals are still preserved, they can significantly influence synaptic dopamine levels (6). Yet again, as in ref. 5, no clear relationship exists between serotonin transporter binding, peak dose dopamine levels, and the development of LID.

Apart from neurotransmitter changes, levodopa may also affect the relationship between resting cerebral blood flow $(\mathrm{CBF})$ and metabolic activity in specific brain regions. $\mathrm{CBF}$ and cerebral metabolic rate for glucose (CMR) are normally coupled (7), such that an increase in metabolic demand will lead to an increase in blood flow $(8,9)$. PD and levodopa treatment, however, each complicate this relationship. 
A
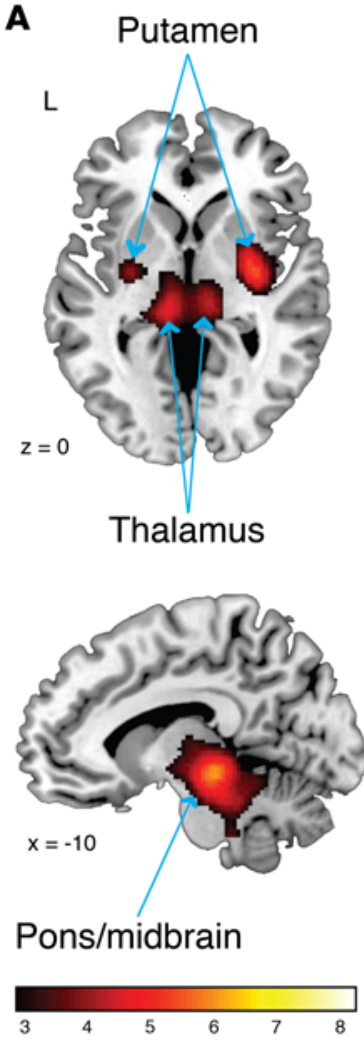

B

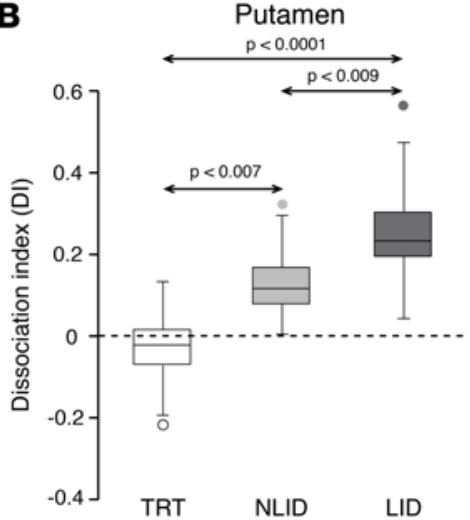

C

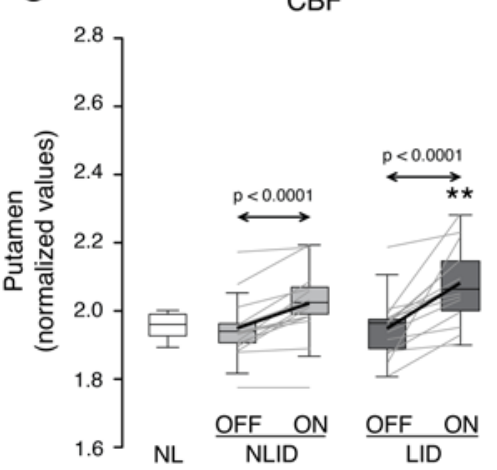

Occipital

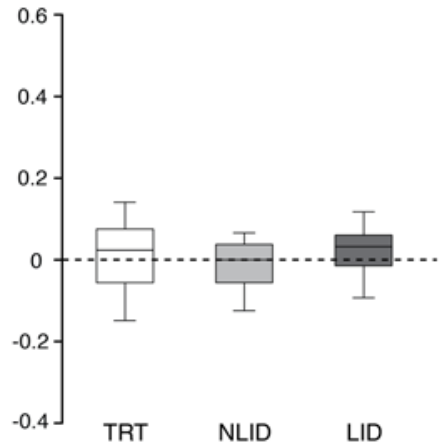

CMR

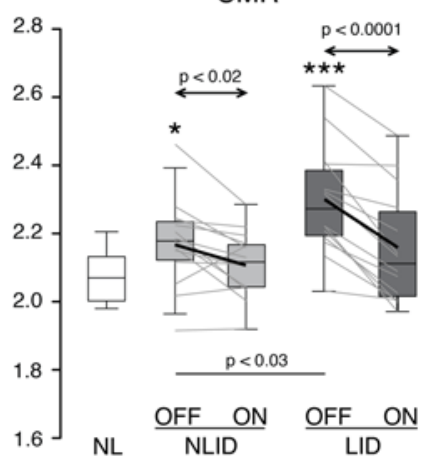

Figure 1. Dissociation of vasomotor and metabolic responses to levodopa in Parkinson's disease subjects with and without levodopa-induced dyskinesias (LID). (A) Scans of cerebral blood flow (CBF) and cerebral metabolic rate (CMR) for glucose were acquired at baseline (OFF) and during levodopa infusion (ON) in 14 Parkinson's disease (PD) subjects with stable motor responses to drug and no dyskinesia (see text). Voxel-wise analysis of the scan data from these non-LID subjects (NLID) revealed significant dissociation of vasomotor ( $\left.\triangle \mathrm{CBF}_{\mathrm{ON}-\mathrm{OFF}}\right)$ and metabolic $\left(\Delta C \mathrm{CMR}_{\mathrm{ON}-\mathrm{OFF}}\right)$ responses to levodopa in the posterior putamen, the internal globus pallidus, the ventrolateral thalamus (top), and the dorsal pons and midbrain (bottom). Clusters were displayed using a red-yellow scale thresholded at $T=3.14(P<0.001$, uncorrected, with cluster extent $>100$ voxels), superimposed on a single-subject MRI template. (B) Dissociation index values ( $\left.\mathrm{DI}=\Delta \mathrm{CBF}_{\mathrm{ON}-\mathrm{OFF}}-\triangle \mathrm{CMR}_{\mathrm{ON}-\mathrm{OFF}}\right)$ were computed for each of the significant regions identified in the NLID analysis. DI values for each region were then computed for the 14 PD subjects who displayed LID during drug infusion, and for the 8 test-retest (TRT) subjects (see text). Of the significant dissociation regions (Table 1), the putamen showed the largest group differences in DI (left). The occipital reference region showed no such changes (right). (C) Regional CBF and CMR values were computed in a volume of interest corresponding to putamen dissociation region identified in the NLID analysis (Table 1). Putamen CBF values (left) measured during levodopa infusion reached abnormally high levels in LID but not NLID subjects. By contrast, CMR values measured in the same region (right) were significantly elevated at baseline in both LID and NLID subjects, declining to normal levels during levodopa infusion. In the box-whisker plots, here and in Figure 3, gray lines denote paired values for individual subjects. Arrows indicate post hoc Bonferroni tests for group comparisons in the 1-way ANOVA (B) or 2-way $2 \times 2$ repeated-measures ANOVA (C). Horizontal bars represent Student's $t$ test between groups. ${ }^{*} P<0.05,{ }^{* *} P<0.01,{ }^{* * *} P<0.001$, Student's $t$ test compared with normal (NL; $\left.n=14\right)$ values.

On the one hand, the dysfunction in the substantia nigra caused by loss of dopaminergic neurons affects resting brain function in interconnected downstream regions, leading to stereotypic metabolic increases in the putamen, globus pallidus, ventral thalamus, and dorsal pons $(10,11)$. This disease-related metabolic pattern, termed PDRP, is highly replicable across patient populations (12-14), and its expression correlates closely with progression (13-15). Levodopa normalizes CMR in these hyperactive nodes of the PDRP network (16), but it also elicits localized increases in CBF at the same nodes (17), likely by stimulating dopamine receptors on the vessel walls or dopamine terminals apposed to capillaries and arterioles $(18,19)$. Our dual-tracer PET study of PD patients showed that, indeed, levodopa infusion increases CBF at the same time it is reducing CMR in the posterior putamen, globus pallidus, ventral thalamus, and dorsal pons (17). In subsequent autoradiographic studies, levodopa produced similar changes in homologous brain regions in the 6-hydroxydopamine (6-OHDA) rat model (20). The pathophysiology underlying this dissociation between vasomotor and metabolic levodopa responses is unknown, and it remains unclear whether the magnitude of dissociation is related the development of LID.

In the current study, we examined the possibility that LID subjects are distinguished by an exaggeration 
A

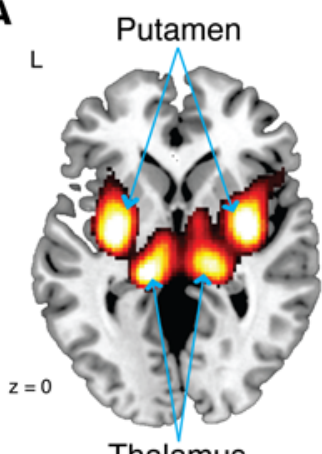

Thalamus

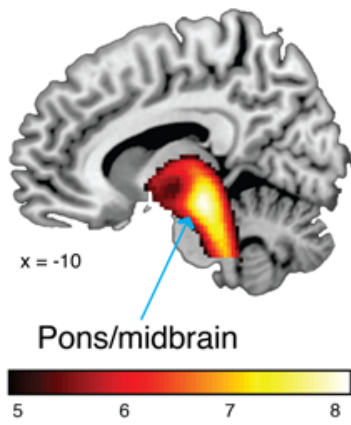

B

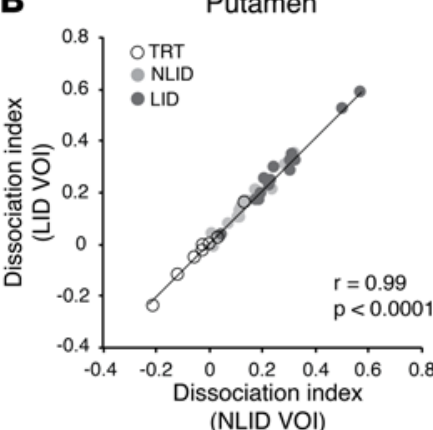

(NLID VOI)

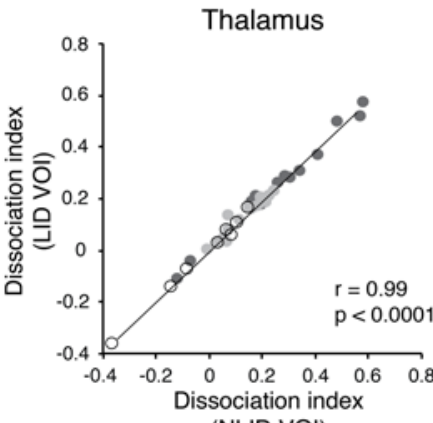

(NLID VOI)

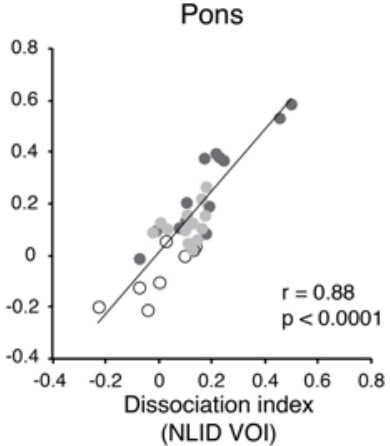

Globus pallidus (internal)

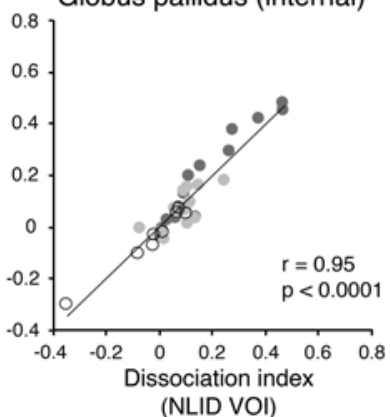

Figure 2. Dissociation of vasomotor and metabolic drug responses in an independent Parkinson's disease sample. (A) We analyzed cerebral blood flow (CBF) and cerebral metabolic rate (CMR) scans acquired ON and OFF levodopa infusion from 14 Parkinson's disease subjects with levodopa-induced dyskinesia (LID) and found significant dissociation of vasomotor and metabolic response to levodopa in regions near those identified in the non-LIDbased (NLID-based) analysis (Figure 1A). Regional dissociation effects identified in the LID-based analysis were generally greater in magnitude and more bilateralized than those seen in their NLID counterparts. Clusters were displayed using a red-yellow scale thresholded at T $=4.50(P<0.05$, family-wise error-corrected, with cluster extent $>100$ voxels), superimposed on a single-subject MRI template. (B) Dissociation index (DI) values were highly correlated ( $r=0.88-0.99, P<0.0001$, regression analysis) for each of the significant dissociation regions identified in the independent LID- and NLID-based analyses. For each of the significant regions identified in the 2 analyses, volumes of interest (VOI) coordinates representing the peak voxel of the corresponding NLID- and LID-based clusters are provided on the $x$ - and $y$-axis labels, respectively. For each region, individual DI values computed in the NLID- and LIDbased VOIs are separately displayed for the 14 LID (dark gray), 14 NLID (light gray), and the 8 test-retest (TRT) (open circles) subjects.

of the regional dissociation effects seen in their non-LID (NLID) counterparts. We used dual-tracer PET to map levodopa-mediated effects on both cerebral blood flow and metabolism at the voxel level, quantified these effects in PD subjects scanned before and during intravenous levodopa infusion, and compared the resulting values for the subjects who manifested LID during drug infusion with those who did not. We also determined whether individual subjects could be distinguished by baseline differences in regional brain function that were present before levodopa administration.

\section{Results}

\section{Dissociation between vasomotor and metabolic responses to levodopa in the putamen distinguishes LID from NLID subjects}

We first interrogated the scan data for regions with significant dissociation of the vasomotor and metabolic responses to levodopa. To minimize possible confounding effects caused by abnormal involuntary movements during imaging, we began by analyzing CBF and CMR scans acquired in NLID subjects ( $n=14: 10$ men and 4 women; age $60.0 \pm 9.9$ [mean \pm SD] years; disease duration $6.1 \pm 5.0$ years), then confirmed results using analogous scan data from their LID counterparts $(n=14: 10$ men and 4 women; age $62.0 \pm$ 9.6 years; disease duration $13.1 \pm 8.4$ years) (see Supplemental Table 1; supplemental material available online with this article; doi:10.1172/jci.insight.86615DS1 and Methods).

Voxel-wise interrogation of scans from individual NLID subjects before and during drug infusion revealed significant levodopa-mediated dissociation effects in the putamen, internal globus pallidus (GP $)_{\mathrm{i}}$, ventrolateral thalamus, and dorsal pons (Figure 1A). The local dissociation index (DI), defined as the difference between 
Table 1. NLID and LID samples: voxel-wise analysis of brain regions with dissociation of vasomotor and metabolic responses to levodopa

\begin{tabular}{|c|c|c|c|c|c|c|c|c|c|}
\hline & \multirow[t]{2}{*}{ Regions } & \multicolumn{3}{|c|}{ Coordinates $^{A}$} & \multirow[t]{2}{*}{$Z \max$} & \multicolumn{3}{|c|}{ Dissociation index (DI) } & \multirow[t]{2}{*}{ Group difference } \\
\hline & & $x$ & $y$ & $z$ & & NLID $(n=14)$ & LID $(n=14)$ & TRT $(n=8)$ & \\
\hline \multicolumn{10}{|c|}{ NLID } \\
\hline & \multirow[t]{2}{*}{ Putamen } & 34 & -8 & -4 & 5.29 & $0.133 \pm 0.025^{t+, \mathrm{D}}$ & $0.270 \pm 0.035^{t t t}$ & $-0.033 \pm 0.036$ & $\begin{array}{c}F_{(2,33)}=18.80 \\
P<0.0001\end{array}$ \\
\hline & & -24 & -10 & 0 & 4.06 & & & & \\
\hline & Thalamus & 14 & -26 & 0 & 4.40 & $0.124 \pm 0.021$ & $0.266 \pm 0.055^{t+t}$ & $-0.022 \pm 0.060$ & $\begin{array}{c}F_{(2.33)}=8.44 \\
P<0.002\end{array}$ \\
\hline & Pons & 10 & -34 & -32 & 4.96 & $0.108 \pm 0.016$ & $0.177 \pm 0.041^{\dagger \dagger}$ & $\begin{array}{c}0.007 \pm \\
0.043\end{array}$ & $\begin{array}{c}F_{(2,33)}=5.27 \\
P<0.02\end{array}$ \\
\hline & $G P_{i}$ & -16 & -6 & -6 & 3.41 & $0.083 \pm 0.020$ & $0.176 \pm 0.043^{\dagger+}$ & $-0.032 \pm 0.050$ & $\begin{array}{c}F_{(2,33)}=6.62, \\
P<0.004\end{array}$ \\
\hline \multicolumn{10}{|c|}{ LID } \\
\hline & Putamen & -32 & -10 & -2 & 7.83 & $0.145 \pm 0.027^{\dagger \dagger}$ & $0.289 \pm 0.037^{t t t}$ & $-0.027 \pm 0.041$ & $\begin{array}{c}F_{(2,33)}=17.67 \\
P<0.0001\end{array}$ \\
\hline & Thalamus & 16 & -24 & 2 & 7.24 & & & & \\
\hline & Pons & -2 & -36 & -38 & 7.47 & $0.121 \pm 0.017^{\dagger \dagger}$ & $0.251 \pm 0.049^{t t t}$ & $-0.072 \pm 0.038$ & $\begin{array}{c}\mathrm{F}_{(2,33)}=15.40 \\
P<0.0001\end{array}$ \\
\hline & $\mathrm{GP}_{\mathrm{i}}$ & 12 & -2 & -12 & 4.91 & $0.071 \pm 0.019$ & $0.196 \pm 0.049^{\dagger \dagger}$ & $-0.043 \pm 0.043$ & $\begin{array}{c}F_{(2,33)}=8.35 \\
P<0.002\end{array}$ \\
\hline
\end{tabular}

${ }^{A}$ Montreal Neurological Institute (MNI) standard space. ${ }^{B}$ Dissociation index $(\mathrm{DI})=\Delta \mathrm{CBF}_{\mathrm{ON}-\mathrm{OFF}}-\triangle \mathrm{CMR}_{\mathrm{ON}-\mathrm{OFF}}$; see text. ${ }^{\mathrm{C}}$-way ANOVA of DI values for the testretest (TRT), non-LID (NLID), and LID patient groups; see text. Values represent $F$ test and $P$ value for the whole model. ${ }^{\circ}$ Values are presented as mean \pm SEM. Bold text denotes regions in which DI differentiates LID from NLID $(P<0.05$, post hoc Bonferroni test). Italicized text denotes regions that show significant levodopa-mediated dissociation ( $P<0.001$, uncorrected) but did not survive the family-wise error (FWE) correction $(P<0.05) .{ }^{+t} P<0.01$, ${ }^{t+\dagger} P<0.001$, post hoc Bonferroni test compared with the TRT group.

the two regional treatment responses $\left(\triangle \mathrm{CBF}_{\mathrm{ON}-\mathrm{OFF}}-\Delta \mathrm{CMR}_{\mathrm{ON}-\mathrm{OFF}}\right)$, differed significantly for the LID, NLID, and test-retest (TRT) groups $\left(\mathrm{F}_{(2,3)} \geq 5.27, P<0.02 ; 1\right.$-way ANOVA) in each of these areas (Table 1$)$, whereas $\mathrm{DI}$ values measured in the occipital reference region did not differ across the 3 groups $(P=0.49$; 1 -way ANOVA) (Figure 1B, right). The putamen exhibited the greatest group differences in local DI values $\left(\mathrm{F}_{(2,33)}=18.80\right.$, $P<0.0001$ ), as well as the largest dissociation effects (Figure 1B, left).

The putamen was, in fact, the only region in which DI differed significantly between LID and NLID subjects: LID subjects showed greater levodopa-mediated dissociation than either the NLID or TRT groups $(P<0.01$ and $P<0.0001$, respectively; post hoc Bonferroni tests) (Figure 1B and Table 1). DI values measured in this region were uniformly positive in both LID and NLID subjects ( 0 of 14 negative values in each of the 2 groups), indicating that vasomotor responses to levodopa consistently exceeded metabolic changes recorded concurrently in the same individuals. In the TRT subjects, by contrast, positive and negative putamen DI values were encountered with similar frequency ( 3 of 8 positive, 5 of 8 negative; $P<0.001 ; \chi^{2}$ tests comparing LID or NLID with TRT). Once again, DI values measured in the occipital reference region did not exhibit the same positive tendency that was observed in the putamen: the frequency of positive values in the LID and NLID subjects (4 of 14 and 7 of 14, respectively) did not differ from that encountered in the TRT group ( 4 of $8, P>0.31 ; \chi^{2}$ tests).

To characterize this pattern further, we separately assessed measurements of local vasomotor and metabolic responses obtained from the putamen dissociation region in each of the subjects. In the baseline unmedicated ("off-state") condition, LID and NLID subjects showed the same mean values for putamen CBF (Figure 1C, left) $(P=0.94$; Student's $t$ test) and did not differ from normal $(P>0.67)$. Levodopa treatment significantly increased putamen CBF values in both LID and NLID subjects (main effect: $P<0.0001$ ); CBF increases were observed in 13 of $14 \operatorname{NLID}(P<0.002)$ and in 14 of 14 LID subjects $(P<0.0003$; 
Table 2. Brain region with increased baseline CBF in LID vs. NLID

\begin{tabular}{|c|c|c|c|c|c|c|c|c|}
\hline \multirow[t]{2}{*}{ Regions } & \multicolumn{3}{|c|}{ Coordinates $^{A}$} & \multirow[t]{2}{*}{$Z \max$} & \multicolumn{3}{|c|}{ Dissociation index (DI) } & \multirow[t]{2}{*}{ Group difference $^{c}$} \\
\hline & $x$ & $y$ & $z$ & & NLID & LID & TRT & \\
\hline SMC & -58 & -18 & 28 & 4.35 & $0.044 \pm 0.017^{\circ}$ & $0.058 \pm 0.021$ & $0.053 \pm 0.029$ & $\mathrm{~F}_{(2,33)}=0.18, P=0.83$ \\
\hline
\end{tabular}

${ }^{A}$ Montreal Neurological Institute (MNI) standard space. ${ }^{B}$ Dissociation index $(\mathrm{DI})=\Delta \mathrm{CBF}_{\mathrm{ON}-\mathrm{OFF}}-\triangle \mathrm{CMR}_{\mathrm{ON}-\mathrm{oFF}}$; see text. ${ }^{\mathrm{C}}$-way ANOVA of DI values for the testretest (TRT), non-LID (NLID), and LID patient groups; see text. Values represent $F$ test and $P$ value for the whole model. ${ }^{D}$ Values are presented as mean \pm SEM. Italicized text denotes regions that show significant levodopa-mediated dissociation $(P<0.001$, uncorrected) but did not survive the family-wise error (FWE) correction $(P<0.05)$.

binomial tests). LID subjects tended toward greater vasomotor response (CBF increases) in the putamen than NLID (interaction effect: $\mathrm{F}_{(1,26)}=3.62, P=0.07$; 2-way repeated-measures ANOVA [RMANOVA]), and their putamen $\mathrm{CBF}$ rose to abnormally high levels in the levodopa-treated "on-state" $(P=0.001$, relative to healthy control values; Student's $t$ test).

We did not find significant correlations between the vasomotor and metabolic levodopa responses $(\triangle \mathrm{CBF}$ and $\triangle \mathrm{CMR})$ in the putamen for the total sample or for the individual LID and NLID groups $(P>0.33)$. In fact, CMR values in this region declined with treatment in both NLID and LID subjects $(P<0.02$ and $P<$ 0.0001, respectively; post hoc Bonferroni tests). In the baseline off-state, CMR values (Figure $1 \mathrm{C}$, right) were abnormally elevated in both LID and NLID subjects $(P<0.0001$ and $P<0.03$, respectively; Student's $t$ tests, relative to healthy control values), with higher values in the former group ( $P<0.03$; Student's $t$ test). On-state putamen CMR was similar in the NLID and LID subjects $(P=0.32$; Student's $t$ test $)$ and did not differ from normal in the former group $(P=0.28)$, though LID subjects showed a marginal abnormality $(P=0.08)$. As with levodopa-mediated vasomotor changes in the putamen, concurrently recorded metabolic responses were highly stereotyped: drug-induced declines in local CMR values were observed in 11 of 14 NLID $(P<0.04)$ and 14 of 14 LID subjects ( $P<0.0003$; binomial tests). Nonetheless, the levodopa-mediated metabolic reduction was greater in LID subjects (interaction effect: $\mathrm{F}_{(1,26)}=6.86, P<0.02 ; 2$-way RMANOVA).

Although off-state putamen $\mathrm{CBF}$ and $\mathrm{CMR}$ values were correlated for the entire infusion cohort (LID and NLID: $r^{2}=0.39, P<0.0005$; linear regression analysis), levodopa disrupted this coupling in

A

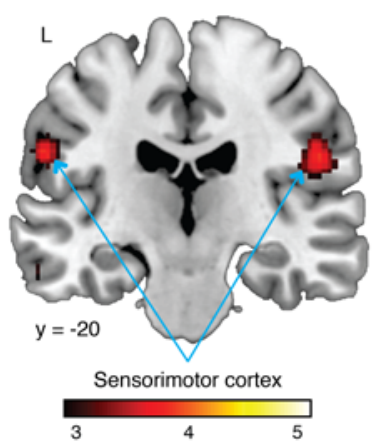

B

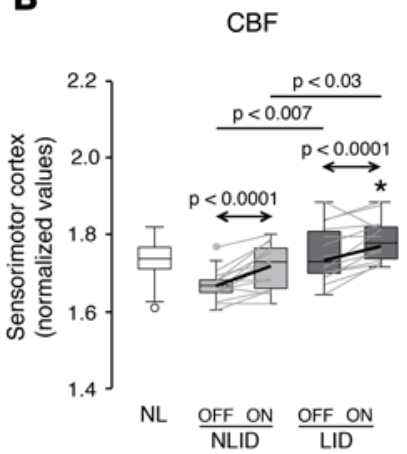

CMR

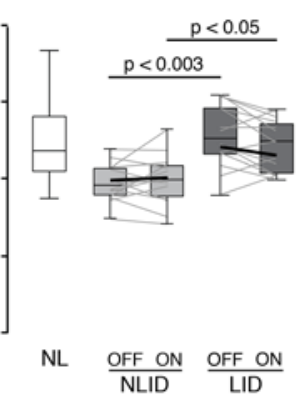

C

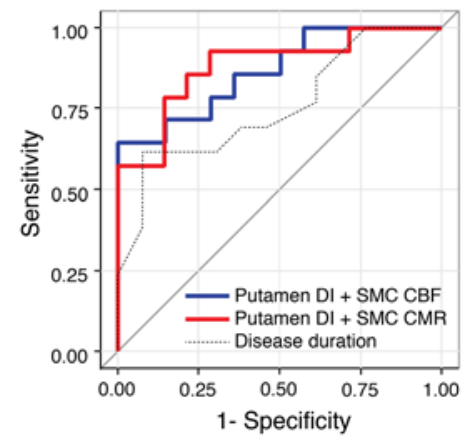

Figure 3. Increased resting activity in the sensorimotor cortex in unmedicated levodopa-induced dyskinesia (LID) and non-LID (NLID) subjects. (A) Voxel-wise comparison of baseline (OFF) cerebral blood flow (CBF) scans from the 14 NLID and 14 LID subjects revealed significantly increased bilateral resting activity in the sensorimotor cortex (SMC) in the latter group. Clusters were displayed using a red-yellow scale thresholded at $T=3.47(P<0.001$, uncorrected, with cluster extent $>100$ voxels), superimposed on a single-subject MRI template. (B) Box-and-whisker plots of the individual subject SMC data. During levodopa infusion (ON), regional CBF values (left) increased significantly in both NLID and LID subjects, whereas cerebral metabolic rate (CMR) values (right) were not altered by drug. Arrows indicate post hoc Bonferroni tests for group comparisons in the 2 -way $2 \times 2$ repeated-measures ANOVA. Horizontal lines represent Student's $t$ tests comparing local values measured in the LID and NLID groups. ${ }^{*} P<0.05$, Student's $t$ test compared with normal (NL; $n=14$ ) values. (C) Receiver-operating characteristic curves showing the discrimination of LID from NLID subjects based upon the combination of putamen dissociation index (DI) and resting SMC CBF (blue curve) or CMR (red curve) values measured in the baseline unmedicated condition. Accurate discrimination of individual subjects was achieved by logistic regression models based on these variables $(P<0.002)$. Each of the 2 -variable discriminant models was superior to a 1-variable model (dotted curve) in which symptom duration was the only predictor $(P<0.02)$. 
Table 3. Summary of levodopa-induced changes in the putamen and sensorimotor cortex

\begin{tabular}{|c|c|c|c|}
\hline & \multicolumn{3}{|c|}{ Putamen } \\
\hline \multicolumn{4}{|c|}{ Levodopa } \\
\hline & OFF & ON & Change \\
\hline CBF & & & Vasomotor \\
\hline NLID & Normal & Marginal elevation & Increase $\mathrm{ftt}^{\dagger t}$ \\
\hline LID & Normal & $\mathrm{High}^{* *}$ & Increase $\mathrm{e}^{t+t}$ \\
\hline CMR & & & Metabolic \\
\hline NLID & High* $^{*}$ & Normal & Reduction $^{\dagger}$ \\
\hline LID & $\mathrm{High}^{* * * \#}$ & Normal & Reduction ${ }^{\dagger \dagger \dagger \# ~}$ \\
\hline CBF/CMR & Correlated & Uncorrelated & Uncoupling ${ }^{\mathrm{A}}$ \\
\hline \multicolumn{4}{|c|}{ Sensorimotor cortex } \\
\hline \multicolumn{4}{|c|}{ Levodopa } \\
\hline & OFF & ON & Change \\
\hline CBF & & & Vasomotor \\
\hline NLID & Normal & Normal & Increase $^{\dagger \dagger}$ \\
\hline LID & Normal”\# & $\mathrm{High}^{* \#}$ & Increase $\mathrm{ftt}^{t+}$ \\
\hline CMR & & & Metabolic \\
\hline NLID & Normal & Normal & No change \\
\hline LID & Normal”\# & Normal" & No change \\
\hline CBF/CMR & Correlated & Correlated & No uncoupling \\
\hline
\end{tabular}

${ }^{*} P<0.05,{ }^{* *} P<0.01,{ }^{* *} P<0.001$ (vs. NL; Student's $t$ test). ${ }^{\dagger} P<$ $0.05,{ }^{t+} P<0.01,{ }^{\text {ttt }} P<0.001$ (ON vs. OFF; paired Student's $t$ test). ${ }^{\#} P<0.05,{ }^{\#} P<0.01$ (LID vs. NLID; Student's $t$ test). ${ }^{A} P<0.05$ (levodopa-mediated change in regression coefficients; Chow test). the corresponding on-state measures $\left(\mathrm{F}_{(2,52)}=14.49, P<0.00001\right.$, Chow test; $r^{2}=0.18, P<0.03$ ) (Supplemental Figure 1). Levodopa-mediated uncoupling in the putamen was significant in both LID and NLID subjects, albeit to a different degree. Uncoupling was significant $\left(\mathrm{F}_{(2,24)}\right.$ $=3.84, P<0.04$; Chow test) in NLID (OFF: $r^{2}=0.31, P<0.04$; ON: $r^{2}=0.19, P=0.12$; linear regression analyses), but it was more pronounced $\left(\mathrm{F}_{(2,24)}=12.40, P<0.0003\right.$; Chow test) in LID (OFF: $r^{2}=0.64$, $P<0.0007$; ON: $r^{2}=0.16, P=0.16$; linear regression analyses).

Validation. We conducted an analogous voxel-wise search in the CBF and CMR scan data from the LID subjects to determine whether the same set of dissociation regions would be identified as in the NLID analysis. Although the LID sample was entirely different from that used in the initial analysis, interrogation of these data revealed significant levodopa-mediated dissociation in areas similar to those identified in the NLID subjects (Figure 2A). The magnitude of dissociation in the LID-derived regions was substantially greater than in the NLID analysis, with bilateralization of the observed effects (Table 1). In each of the significant dissociation regions, DI values computed in volumes of interest (VOIs) defined by LID- or NLID-based spatial coordinates (see Methods) correlated closely across the PD sample ( $r>0.87, P<0.0001$; Pearson's correlation) (Figure $2 \mathrm{~B})$. The convergence of these 2 independent analyses underscores the stability of the observed levodopa-mediated dissociation effects.

\section{Baseline differences in resting cerebral function in the SMC distinguish LID and NLID}

We next determined whether LID and NLID subjects were distinguishable by differences in resting cerebral activity in the medication-free baseline condition. Voxel-wise comparison of baseline CBF scans from the LID and NLID subjects disclosed significant differences in the right and left sensorimotor cortex (SMC) (Figure 3A), a region associated with somatotopic representation of the lower face and neck (Table 2). LID subjects showed higher CBF values in this region in the off-state than NLID subjects $(P<0.007$; Student's $t$ test) (Figure 3B, left). Levodopa infusion increased CBF in both the LID and NLID subjects (main effect: $P<0.0001$ ) and to a similar degree (interaction effect: $\mathrm{F}_{(1,26)}=0.76, P=0.39$; 2-way RMANOVA). Levodopa-mediated CBF increases in the SMC were observed in 11 of 14 NLID and in 12 of 14 LID subjects ( $P<0.04$ and $P<0.008$ for the 2 groups, respectively; binomial tests), with CBF values higher in LID than in NLID subjects $(P<0.03$; Student's $t$ test); NLID CBF values did not differ from normal $(P=0.85$ ) (Figure 3B, left). Baseline CMR values in the SMC were also higher in LID than NLID subjects $(P<0.003$; Student's $t$ test), but levodopa did not produce significant local changes in CMR values (Figure 3B, right) in either LID or NLID subjects $(P>0.45)$.

These findings can be summarized as follows (Table 3). Levodopa increased local CBF in both the putamen and SMC, attaining abnormally high on-state levels in LID but not in NLID subjects. Metabolic responses in the 2 regions, however, differed: levodopa decreased CMR in the putamen but not in the SMC. Moreover, levodopa altered the relationship between CBF and CMR in the 2 regions. In the putamen, levodopa produced enormous dissociation, leading to uncoupling (metabolism diminished while blood flow increased). In the SMC, CBF and CMR remained coupled (metabolism stayed the same, while blood flow increased; SMC baseline correlation: $r^{2}=0.23, P=0.01$; on levodopa: $r^{2}=0.28, P<0.004$ ).

\section{Increased resting SMC activity is associated with cortical dopaminergic deafferentation} In $\mathrm{PD}$, increased metabolic activity in the putamen is associated with loss of dopaminergic projections to this region from the substantia nigra beginning at early disease stages $(21,22)$. We therefore considered the possibility that, by analogy, the higher baseline SMC activity in LID subjects might be linked to local loss of mesocortical dopaminergic afferents as occurs with advanced disease (23).

The integrity of dopaminergic afferent projections in a given region can be determined in vivo using $\left[{ }^{18} \mathrm{~F}\right]$ fluorodopa (FDOPA) PET to map the relevant terminals. Presynaptic dopaminergic imaging with this 

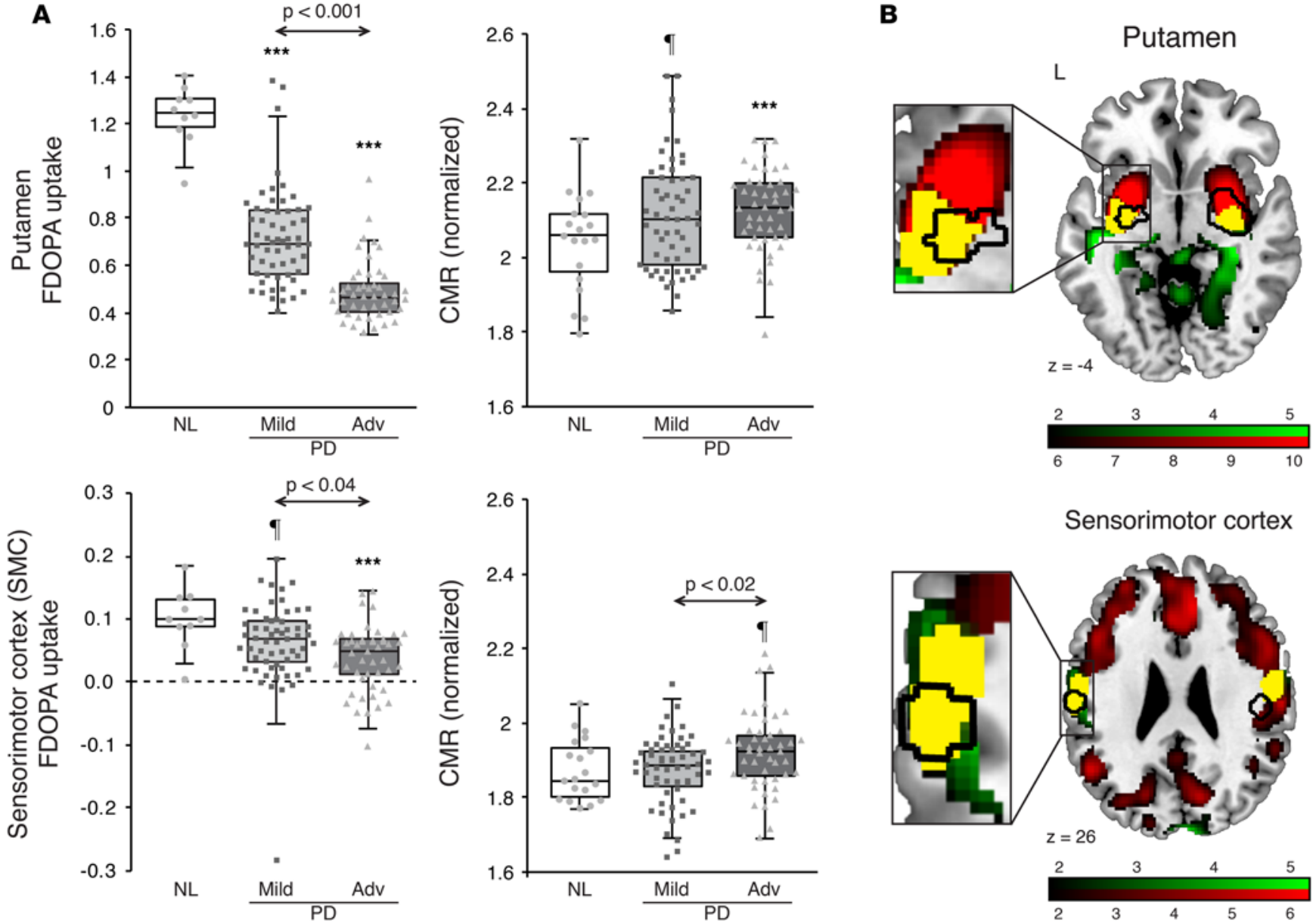

Figure 4. Colocalization of levodopa-mediated cerebral blood flow (CBF) responses and dopaminergic deafferentation in the putamen and sensorimotor cortex of subjects with advanced Parkinson's disease (PD). (A) Left: $\left.{ }^{18} \mathrm{~F}\right]$ fluorodopa (FDOPA) uptake was significantly reduced in the putamen (top) in both the 59 mild (light gray) and 47 advanced (dark gray) PD subjects, and in the sensorimotor cortex (SMC) (bottom) in the advanced patients, compared with 10 healthy control (NL) subjects. Right: In advanced PD subjects, cerebral metabolic rate (CMR) values were significantly higher than normal in the putamen ( $P$ $<0.001$; Student's $t$ test) but only marginally increased in the SMC $(P=0.08)$, compared with $19 \mathrm{NL}$ subjects. Arrows indicate Student's $t$ tests between the 2 PD groups. " $P<0.10$, ${ }^{* * *} P<0.001$, Student's $t$ test compared to NL values. Note the outlier with extremely low FDOPA uptake in the SMC (left, bottom); excluding this subject did not alter the significance of the group comparisons. (B) Regions with significant reduction in dopaminergic input (identified in a voxel-wise analysis of FDOPA PET scans from 47 advanced PD subjects) are denoted in red. Similarly, regions with significant CMR elevation (identified by voxel-wise analysis of $\left.{ }^{18} \mathrm{~F}\right]$ fluorodeoxyglucose PET scans acquired in the same individuals) are denoted in green (see text). Areas of overlap (yellow) exhibit both dopaminergic deafferentation and increased local metabolic activity. In the current study, areas of dissociation (black contours) in the putamen (top) and areas of increased baseline CBF in the SMC (bottom) colocalized almost exclusively with areas of overlap (yellow) (insets). Maps of reduced FDOPA uptake were thresholded at $T=6.0, P<0.001$, uncorrected, for the putamen (top) and at $T=1.67, P<0.05$, uncorrected, for the SMC (bottom). Maps of increased CMR were thresholded at $T=1.67, P<0.05$, uncorrected, for both regions. Displays were superimposed on a standard MRI template.

radiotracer has the added advantage of mapping DOPA decarboxylase (DDC) activity in dopaminergic and serotonergic terminals throughout the brain (24-26). Given that DDC converts exogenous levodopa to dopamine in the brain, voxel-wise analysis of FDOPA uptake over the whole brain also provides a means of relating levodopa-mediated changes in regional CBF and/or CMR to the underlying capacity for dopamine production (17). Because of dosimetric limitations, PET imaging with additional radiotracers to evaluate presynaptic dopaminergic function was not possible in the previously mentioned PD subjects, so we analyzed combined dopaminergic and metabolic brain imaging data from a large independent sample of $106 \mathrm{PD}$ patients ( 68 men and 38 women; age $57.0 \pm 11.3$ years) from Cologne, Germany (see Supplemental Table 2 and Methods). These data were used to determine, first, whether the SMC region that was identified above is a target of afferent dopaminergic projections; and second, whether the increase in SMC metabolic activity was associated with disease-related loss of dopaminergic input to the same brain region. To this end, we analyzed imaging data from 59 mildly affected (Hoehn and Yahr stages 1-2) and 47 more 


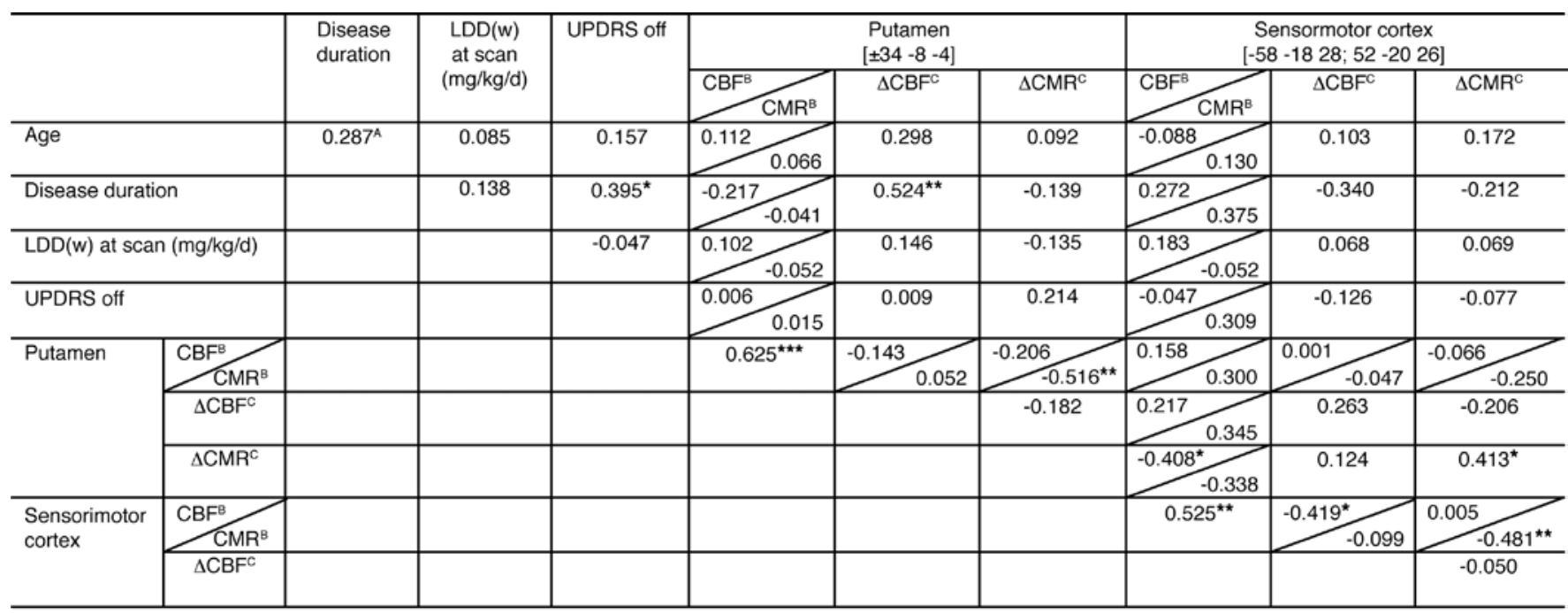

Figure 5. Correlations of demographic, clinical, and imaging data. ${ }^{A} P e a r s o n$ product-moment correlation coefficient. ${ }^{B} B a s e l i n e ~(o f f-s t a t e) ~ C B F$ and $C M R$ values. ${ }^{C} \triangle C B F / \triangle C M R$ represent $[O N-O F F]$ differences for CBF and CMR with levodopa infusion. Significant correlations at ${ }^{*} P<0.05$, ${ }^{*} P<0.01,{ }^{* *} P<0.001$. LDD(w), weight-corrected levodopa daily dose. UPDRS, Unified Parkinson's Disease Rating Scale motor ratings.

advanced (Hoehn and Yahr stages 3-4) PD subjects who were scanned both with FDOPA PET, to quantify the functional integrity of dopaminergic afferent projections, and with $\left[{ }^{18} \mathrm{~F}\right]$ fluorodeoxyglucose (FDG) PET, to measure off-state CMR values in the putamen and SMC regions identified above (see Methods).

FDOPA uptake in the putamen and SMC was greater than corresponding occipital lobe reference values measured in the PD and healthy control groups $(P<0.0001$; paired Student's $t$ tests). Subjects with both mild and advanced PD showed lower putamen FDOPA uptake (Figure 4A, top left) than healthy subjects $(P<0.0001$; Student's $t$ tests); the advanced PD patients had even lower values than their mild PD counterparts $(P<0.001)$. It is worth noting that FDOPA uptake in the SMC was an order of magnitude lower than corresponding putamen measures from the same subjects (Figure 4A, bottom left). The reduction in SMC FDOPA uptake observed in mild PD was marginal relative to healthy subjects ( $P=0.08$; Student's $t$ test). These changes were significant, however, in advanced PD relative to both healthy subjects $(P<0.001)$ and to mildly affected patients $(P<0.04)$. Using the FDG PET scans acquired in these subjects, we computed CMR values for the identical brain regions. For the putamen (Figure 4A, top right), CMR values were marginally greater than healthy subjects in mild $\mathrm{PD}(P=0.07$; Student's $t$ test $)$ and significantly elevated in advanced PD patients $(P<0.01)$. For the SMC (Figure 4A, bottom right), advanced PD subjects showed significantly higher CMR than their mildly affected counterparts $(P<0.02$; Student's $t$ test).

These findings associate the modest CMR increases seen in the SMC of LID subjects with loss of mesocortical dopaminergic projections to the same brain region. Reductions in FDOPA uptake in this region were significant only in more advanced patients, representing a decline of $60 \%-65 \%$ from the normal mean, consistent with only partial loss of DDC activity in the SMC even in these cases. Indeed, with 35\%-40\% of normal PET signal remaining in this region, it is likely that there is still sufficient DDC activity to allow for local production of dopamine from exogenous levodopa in individuals with advanced disease. This point is substantiated by the spatial correspondence between the areas of the putamen and SMC that were identified as having significant local vasomotor responses to levodopa (Figure $4 \mathrm{~B}$, black contours) and those with residual FDOPA uptake (Figure 4B, red). Thus, the levodopa-mediated vasomotor responses observed in the putamen and SMC colocalized with areas of significant though incomplete dopaminergic denervation that were independently identified in the same brain regions. Apart from encompassing dopaminergic projection targets in the putamen and SMC, areas identified by voxel-wise analysis also colocalized with regions of increased baseline metabolic activity (Figure 4B, green). Indeed, both of the areas that were found to mediate LID (i.e., those bounded by the black contour lines in Figure 4B) were characterized by spatial overlap (Figure 4B, yellow) between zones of partial dopaminergic denervation and increased local metabolic activity. In aggregate, the data suggest that in the putamen, both abnormalities develop early in the disease course and worsen over time. In the SMC, by contrast, these changes appear only in advanced disease. 
A

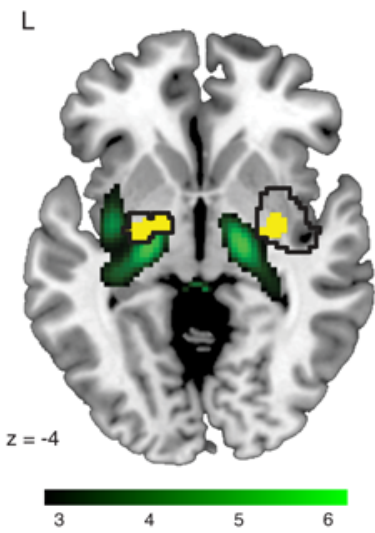

C

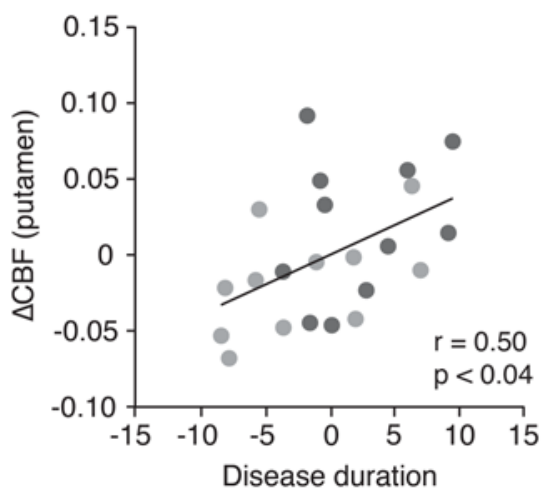

B

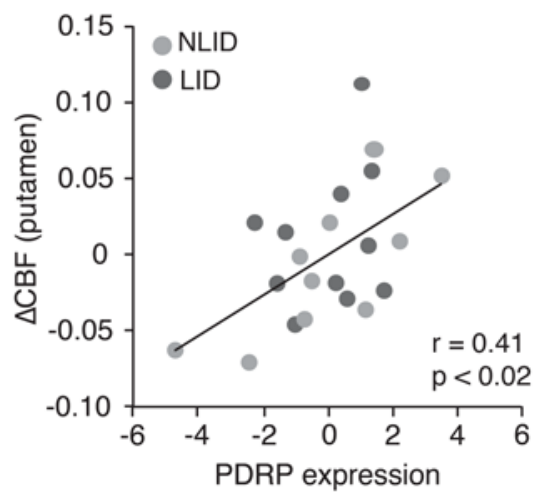

D

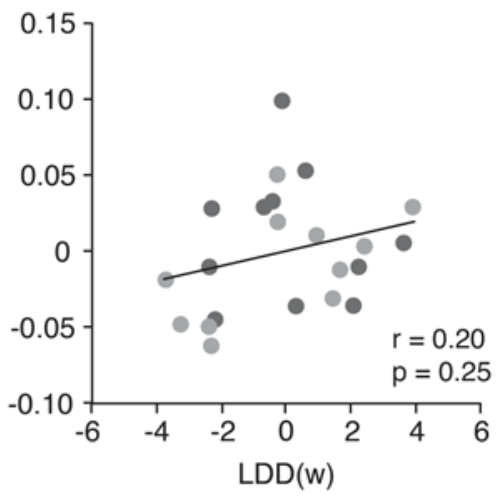

Figure 6. Clinical and network correlates of the putamen vasomotor response to levodopa. Multiple regression analysis was performed to evaluate the relationship between vasomotor levodopa response $\left(\triangle \mathrm{CBF}_{\mathrm{ON}-\mathrm{OFF}}\right)$ measured in the putamen dissociation region and independent descriptors of disease progression and chronic levodopa exposure (see Methods). In addition to disease duration and weight-corrected levodopa daily dose $[\operatorname{LDD}(w)]$, the model included offstate expression of the PD-related metabolic covariance pattern (PDRP), a metabolic network descriptor of disease progression measured over the entire brain (see text). (A) There was a striking spatial overlap (yellow) between PDRP regions with relatively increased metabolic activity (green) and areas with significant levodopa-mediated dissociation effects in the putamen (black contours). Positive voxel weights on the PDRP topography were thresholded at $\mathrm{T}=3.12, P<0.001$, uncorrected, for the putamen. The display was overlaid on a standard MRI template. (B-D) Partial correlation leverage plots of the combined levodopa-induced dyskinesia (LID) (dark gray) and non-LID (NLID) (light gray) sample ( $n=11$ each) illustrate the unique effect of each predictor on the putamen vasomotor response to levodopa. (B) In accord with the observed spatial overlap, off-state PDRP expression exhibited a significant independent relationship with the putamen vasomotor response. (c) There was a significant relationship between the local dopaminergic vasomotor response and disease duration. (D) Levodopa dose $[\operatorname{LDD}(w)]$ was not a significant predictor of putamen $\triangle \mathrm{CBF}$.

\section{Clinical correlates of regional dissociation effects}

Discriminating LID from NLID. Can the dissociation effects in the putamen and differences in baseline activity in the SMC discriminate LID subjects from their NLID counterparts? We constructed a hierarchical set of logistic regression models to answer this question. The models were based on (a) local CBF and CMR values measured at baseline and during drug infusion, (b) vasomotor and metabolic treatment responses, and (c) DI values. For each region (putamen and SMC), the measures were evaluated as single classifiers and were ranked in order of discrimination accuracy. Then, using the combination of single measures that provided the best LID versus NLID discrimination for each region, we tested the ability of the model to correctly classify individual subjects.

Of the individual regional classifiers that we tested, the most accurate discrimination between LID and NLID subjects was provided by putamen DI and baseline SMC CBF values $(P<0.005$ for each measure; likelihood ratio tests), accounting for $39.9 \%$ and $34.0 \%$ of the variance in the classification data, respectively. We substantially improved overall classification accuracy by entering both measures into one predictive model, which accounted for $51.1 \%$ of the variance in the data $(P<0.002$; likelihood ratio test) and was reflected in the receiver-operating characteristic (ROC) analysis, with an area under the curve (AUC) of 0.87 (Figure 3C, blue curve). Similarly high classification accuracy was achieved by combining putamen DI with baseline SMC CMR: these two regional measures together accounted for $56.0 \%$ of the classification variance ( $P<0.001$; likelihood ratio test), with an AUC of 0.88 on ROC analysis (Figure $3 C$, red curve). Thus, the combination of baseline SMC activity (either CBF or CMR) and putamen DI accurately discriminated LID from NLID subjects. Although disease duration alone was also significant as a classifier $(P<$ 0.02 ; likelihood ratio test; Figure 3C, dotted curve), it explained only $28 \%$ of the classification variance in the data, with an ROC AUC of 0.75 .

Correlation with disease measures. We next examined the relationship between levodopa-mediated regional responses in the putamen and SMC and independent clinical measures of disease progression. Individual subject $\mathrm{CBF}$ and $\mathrm{CMR}$ values for the 2 regions measured on- and off-levodopa, and corresponding regional vasomotor and metabolic responses to drug, were separately correlated with disease duration, baseline 
(off-state) motor disability ratings, and levodopa daily dose at the time of the study. We found significant correlations between disease duration and putamen $\triangle \mathrm{CBF}(r=0.52, P<0.01$; Pearson's correlation), as well as putamen DI ( $r=0.43, P<0.05)$ (Figure 5). That said, levodopa-mediated vasomotor responses in the putamen did not correlate with baseline motor disability ratings or with levodopa daily dose $(P>0.23)$. There were no significant correlations between levodopa-mediated vasomotor responses in the SMC and clinical measures, although a trend-level relationship was observed with disease duration $(P=0.08)$. No clinical correlations with local metabolic responses to levodopa were observed for either brain region $(P>0.28)$.

We noticed that in the putamen, areas of significant levodopa-mediated dissociation and increased vasomotor drug response (Figure 6A, black contours) colocalized (yellow) with the metabolically active core regions (green) of the abnormal PD-related network topography (PDRP) $(12,13)$. Because PDRP expression constitutes an objective measure of disease progression at the network level, we added this measure to disease duration and weight-corrected levodopa daily dose $[\mathrm{LDD}(\mathrm{w})]$ to create a predictive model of the vasomotor response to levodopa in the putamen; this analysis was performed in the 22 (of 28) PD subjects for whom these clinical measures were available. Whereas disease duration and LDD $(\mathrm{w})$ together accounted for a mere $25 \%$ of the variance in the putamen vasomotor response to levodopa $(P=0.06$; multiple regression analysis), adding PDRP expression to the model improved the prediction of putamen $\triangle \mathrm{CBF}$ by $21 \%$. In fact, the 3 predictors together explained $46 \%(P<0.01)$ of the variance in putamen vasomotor response. We performed partial correlation analysis to determine the effect of each predictor on the regional response measure after excluding the effects of the other predictors. The results suggest that the vasomotor response to levodopa in the putamen is linked to independent effects of disease duration $(r=0.50 . P<$ 0.04; leverage plot) and PDRP expression $(r=0.41, P<0.02)$, but not to weight-corrected levodopa dose $(r$ $=0.20, P=0.25$ ) (Figure $6, \mathrm{~B}-\mathrm{D})$. By contrast, none of these measures predicted $\triangle \mathrm{CMR}$ in the putamen, singly or in combination $(P=0.80)$.

\section{Discussion}

Functional anatomy of LID. We observed reproducible dissociation of vasomotor and metabolic responses to levodopa involving the putamen, globus pallidus, ventrolateral thalamus, and dorsal pons/midbrain in LID and NLID subjects. The response to levodopa in each of these regions was highly stereotyped, with few if any violations in either of the 2 groups. The LID subjects were distinguished, however, by the combination of exaggerated levodopa-mediated dissociation in the putamen and baseline differences in resting SMC activity.

Analysis of individual CBF and CMR values suggested that the prominent LID-related dissociation observed in the putamen stemmed from relative increases in the local vasomotor drug response. In accordance with our previous findings in NLID subjects $(16,27)$, levodopa consistently lowered the abnormally high off-state regional CMR values in the putamen in members of both LID and NLID groups. This contrasted with the concurrent vasomotor changes in the same region, in which levodopa drove baseline $\mathrm{CBF}$ to higher on-state values, reaching abnormally elevated levels in LID. Indeed, in the baseline off-state, putamen CBF values did not differ from normal in either LID or NLID groups, but on-state measurements exceeded the upper limit of normal in approximately $75 \%$ of the LID subjects. We note, however, that similar on-state elevations were also present in 50\% of the NLID subjects. The individual data therefore indicate that an increase in the putamen vasomotor response to levodopa in a given subject does not guarantee the presence of LID, even if CBF rises to abnormally high levels. Analogously, a reduction in putamen CMR does not predict LID at the individual subject level.

In fact, our data link LID to the presence of additional downstream cortical changes in the sensorimotor cortex: the distinction between LID and NLID relied on differences in resting SMC activity that are present at baseline before administration of the drug. This finding suggests that the abnormal involuntary movements defining LID are facilitated by localized increases in motor cortical activity, which develop with advancing disease. The results of the step-wise logistic regression model (Figure 3C) support this interpretation.

It is worth noting that levodopa induced involuntary movements of the face and neck in virtually all of the LID subjects who participated in the current study. This is consistent with the somatotopic localization of the SMC cluster that was identified by direct voxel-wise comparison of maps of baseline CBF and CMR from the LID and NLID groups and reflects the somatic distribution of LID symptoms in PD patients $(28,29)$.

Relationship between levodopa-mediated changes in regional brain function and local dopaminergic input. We observed a significant correlation ( $r=0.41, P<0.05$; Pearson's correlation) between the $\triangle \mathrm{CMR}$ in the putamen and SMC (Figure 5). Given the known relationship of local CMR values to underlying synaptic 
activity $(30,31)$, this correlation is consistent with substantial anatomical and functional interconnections between the 2 regions. Indeed, both the putamen and SMC exhibited significant relationships between baseline metabolic activity and dopaminergic input in the large independent (German) sample of PD patients and control subjects who underwent dual-tracer PET. These data revealed substantial reductions in nigrostriatal dopaminergic function for the putamen VOI (Figure 4A, top left) in mildly affected PD subjects (Hoehn and Yahr stages 1-2) and even greater reductions in advanced PD (Hoehn and Yahr stages 3-4). These changes, along with the concurrent increases in local metabolic activity (Figure 4A, top right), are consistent with findings from an independent longitudinal cohort of early-stage PD patients (21). Analogous changes were seen in the SMC, albeit later in the disease process than in the putamen. Dopaminergic input to the SMC, represented by local FDOPA uptake, was an order of magnitude lower than analogous measurements from the putamen obtained in PD and control subjects (23). The 2 regions also differed in the relative reductions in local FDOPA uptake that were observed for patients with mild and advanced PD. Advanced PD subjects exhibited significant loss of dopaminergic input to the SMC, with concomitant increases in local metabolic activity. In the putamen, by contrast, substantial changes in both measures were present with early as well as more advanced disease.

Precise knowledge of LID versus NLID status was not available for the Cologne sample. Even so, we did note a striking clinical similarity between members of the advanced PD reference group scanned at Cologne and the LID subjects in our clinical sample. This allowed us to consider how local dopaminergic denervation and concomitant metabolic changes relate to the appearance of LID. FDOPA uptake fell to subnormal levels in both the putamen and the SMC, with significant reductions occurring earlier in the disease course for the former. The loss of PET signal was far from complete, however: uptake values approximating $40 \%$ of normal were observed in both regions, even in the more advanced patients. The substantial amount of FDOPA uptake remaining in these areas, mainly reflecting residual DDC activity $(23,25)$, is compatible with greater levels of local serotonergic innervation that have been posited to occur in LID (6, 32). The current data suggest that even at later stages of disease, sufficient amounts of DDC activity are present in the putamen and SMC to convert exogenous levodopa to dopamine, thereby giving rise to the increases in local CBF that we observed during drug infusion in both regions.

Although LID subjects exhibited elevations in on-state CBF values in both the putamen and SMC, the mechanism underlying these changes is likely different for the two regions. In the putamen, baseline $\mathrm{CBF}$ was similar for LID and NLID (and normal) subjects, but the response to levodopa $\left(\triangle \mathrm{CBF}{ }_{\mathrm{ON}-\mathrm{OFF}}\right.$ ) was comparatively greater in LID. In the SMC, by contrast, $\mathrm{CBF}$ values were higher at baseline in the LID subjects, but $\triangle \mathrm{CBF}_{\mathrm{ON}-\mathrm{OFF}}$ was similar for the 2 groups. The reason for this disparity is unclear, though off-state CBF tended to be lower in the NLID subjects in the SMC (Figure 3). Reduction in dopaminergic input to the putamen and SMC, with persistent upregulation of dopamine D1 receptors and concomitant supersensitivity of downstream signaling, is considered critical for the development of LID $(33,34)$. This is consistent with the dopamine terminal loss in the advanced PD (German) cohort, which was similar for the 2 regions (putamen: $-61.5 \%$; SMC: $-60.7 \%$; Figure 4A, left panels). Yet the data suggest that the putamen and SMC differ in the rate at which dopaminergic deafferentation and concomitant downstream functional changes develop in each region. Indeed, nigrostriatal dopaminergic dysfunction is already well established in early PD, accompanied by progressive increases in putamen metabolic activity (21). Degeneration of dopaminergic afferent projections is comparatively slower for the mesocortical system. Significant loss of dopaminergic input to the SMC and concomitant metabolic increases in this region become evident only at more advanced stages of the disease. Thus, in advanced PD, with symptom duration of 10 years or more, significant loss of dopaminergic input is seen in both the putamen and SMC, along with increased local metabolic activity. In the putamen, metabolic overactivity subsequent to dopamine deafferentation is present at the time of diagnosis (21), whereas in the SMC such changes may not become evident until after an additional 6-8 years of symptoms. It is worth noting that the 2 regions also differed in their metabolic responses to levodopa, with significant treatment-mediated reductions in abnormally high baseline CMR values in the putamen, but no parallel change in SMC values (which were close to normal range to begin with). These metabolic differences likely reflect local neuronal features rather than vascular effects.

Pathogenesis of increased vasomotor response to levodopa in LID subjects. Our results accord with findings from human PD studies and experimental models (4) indicating that both chronic levodopa exposure and loss of striatal dopaminergic input are essential for the development of LID (4). Nonetheless, the exaggeration of levodopa-mediated dissociation that we observed in the putamen of LID subjects, with local 
uncoupling of CBF and CMR, suggests that changes in neurovascular regulation are also involved.

It is tempting to relate these changes to underlying angiogenesis, with localized endothelial proliferation in basal ganglia regions subsequent to chronic levodopa exposure (35-37). As noted above, long-term loss of dopaminergic projections to this region in awake unmedicated PD subjects is associated with sustained increases in local metabolic activity. Escalating tissue energy demand in denervated brain regions could cause local upregulation of VEGF, with consequent angiogenesis (38). Resting-state coupling of local CBF and CMR would likely be maintained under these circumstances, provided that vascular tone is not perturbed by hypercapnia (39) or by the administration of vasoactive agents (40). Vascular growth can also be facilitated by mechanical conditions such as intraluminal shear stress, as can occur in the setting of increased blood flow to the tissue (38). Thus, angiogenesis in the putamen might be promoted by increases in local $\mathrm{CBF}$ that take place in response to levodopa. Nevertheless, levodopa influx is not determined solely by $\mathrm{CBF}$ (41); alterations in blood-brain barrier (BBB) permeability are also likely to be relevant. Indeed, in the experimental LID model, angiogenesis is accompanied by the formation of immature blood vessels with localized BBB leakage in the basal ganglia $(20,37)$. Interestingly, in this area significant BBB breach is seen only in animals studied after receiving levodopa. This suggests a possible mechanistic relationship between levodopa-mediated changes in basal ganglia perfusion and BBB integrity on the one hand and concurrent production of LID on the other (20). It is interesting to note that BBB disruption in the putamen has been reported in postmortem samples obtained from individuals with PD (42). Whether analogous changes are present outside the basal ganglia, particularly in cortical motor regions, is a topic of ongoing investigation.

There are currently no imaging tools to demonstrate angiogenesis in the brains of living subjects. Even so, the current findings provide an important insight into the relationship between the observed levodopa-mediated vasomotor responses and the underlying disease process over the long term. In this study, disease measures were tested as possible predictors of the putamen vasomotor response if they were associated with local angiogenesis in the rat model $(35,36)$ and/or were related to the development of LID in human PD subjects $(37,43)$. Thus, symptom duration (a rudimentary descriptor of disease progression) and the weight-corrected levodopa daily dose (a correlate of overall levodopa exposure; see Methods) were chosen as clinical measures to be tested in a multivariate explanatory model. Based on the above, we hypothesized that chronic disease-related increases in local metabolic activity promote angiogenesis in selected brain regions. This possibility was supported by the observation that areas with significant dissociation effects were localized to the metabolically active components of the PDRP topography (17). The vasomotor response to levodopa in the putamen correlated significantly with symptom duration, even after correction for subject differences in the weight-corrected levodopa daily dose, and was independent of drug dose, whether or not corrected for differences in symptom duration. In this respect, human PD appears to differ from the 6-OHDA rodent model, wherein both angiogenesis and the development of LID depend on levodopa dose and the length of time the animal was exposed to the drug after lesioning $(36,37)$. Nonetheless, the current findings in human PD subjects accord well with a recent epidemiological study in which LID was associated more with symptom duration than with the length of levodopa treatment (43).

In addition to symptom duration, the vasomotor response to levodopa in the putamen correlated with concurrent off-state PDRP expression measured at the time of PET. Expression values for this disease-related metabolic network $(12,13)$ can be seen at the earliest stages of the disease, predating the appearance of clinical signs by several years $(21,44,45)$. We have found that in individual subjects, PDRP expression increases at a stable rate over the first 10-12 years following diagnosis $(13,15)$, progressively "crowding out" the normal network topographies that dominate the healthy resting state (46). The distinctive topography of the PDRP provides a link between increased local metabolic demand and compensatory angiogenesis on the one hand and the regional vasomotor response to levodopa on the other. The data suggest that the regions with significant dissociation effects were defined by two shared features: (a) they were included within the space encompassed by the metabolically active core nodes of the PDRP network; and (b) they were the targets of $\mathrm{DDC}^{+}$projections from the dopaminergic substantia nigra and/or serotonergic raphe nuclei. These common characteristics were visualized in the Cologne reference sample as the spatial overlap of areas with increased metabolic activity (FDG PET) and reduced local DDC expression (FDOPA PET) in the putamen and SMC (Figure 4B). Indeed, the significant LID-related clusters identified in The Feinstein Institute sample by voxel-wise analysis of CBF and CMR scans from the same subjects (Figure $4 \mathrm{~B}$, black contours) were composed almost entirely of regions that exhibited both of these features (Figure $4 \mathrm{~B}$, yellow). 
Based on these observations, we can relate the prominent regional dissociation effects seen in LID to localized disease-related metabolic changes occurring at the network level. It is conceivable that the chronic increases in PDRP expression that occur with disease progression drive the development of angiogenesis over the long term in metabolically active network regions. Thus, compensatory angiogenic effects are likely to be most prominent in PDRP areas such as the putamen/globus pallidus, which have the highest levels of metabolic activity in the unmedicated resting state. Angiogenesis in these regions can also be promoted by local dopamine D1 receptor stimulation, as has been observed in the 6-OHDA rat model (36). To date, however, no consistent relationship between levodopa-mediated vasomotor responses and drug dose has been documented. Detailed assessments of cumulative doses of levodopa and dopamine agonist drugs will be needed to determine the relevance of this factor to the development of LID in human PD subjects.

How can the results of this study be used to address this vexing side effect of levodopa treatment and the underlying neurovascular pathology? Selective D1 antagonist drugs could be used to block the acute vasoactive effects of levodopa $(4,36)$; non-dopaminergic agents such as $\alpha 1$ adrenergic antagonists (47) and nicotine (48) have also been suggested for the symptomatic treatment of LID. In addition to having potent synaptic effects in the denervated striatum, these drugs are locally vasoactive (19), and may act through both microvascular and neuronal mechanisms. Because angiogenesis likely represents an adaptation to the progressive increases in local metabolic demand (35-37) consistently found in core PDRP network regions (11, 21), VEGF inhibitors could, in theory, delay or block the onset of LID in chronically levodopa-treated PD patients. Given the strong baseline coupling of $\mathrm{CBF}$ and $\mathrm{CMR}$, however, which supports the notion that angiogenesis is an adaptive response to the local metabolic demands imposed by the disease itself, this course may be unwise: chronic VEGF inhibition might hinder this adaptation and adversely affect long-term tissue viability.

A more effective means of reducing hypermetabolism-driven angiogenesis would be subthalamic nucleus (STN) deep brain stimulation (DBS). Studies have consistently shown that STN DBS reduces PDRP expression $(13,16,49,50)$; in fact, STN DBS alleviates symptoms of parkinsonism in proportion to the degree of PDRP modulation that is achieved $(16,50)$. More to the point, the motor STN is anatomically and functionally linked to the posterior putamen, globus pallidus, and SMC (31), and high-frequency STN stimulation lowers resting metabolism in these regions $(16,50,51)$, likely through antidromic modulation of the corresponding projection pathways (52). If sustained hypermetabolic activity over a period of years drives angiogenesis in PD, it is conceivable that earlier initiation of STN stimulation could correct network-related hypermetabolism and prevent LID from developing in the first place.

\section{Methods}

Subjects. We studied $28 \mathrm{PD}$ subjects (20 men and 8 women; age $61.0 \pm 9.6$ [mean $\pm \mathrm{SD}$ ] years; duration $8.7 \pm 9.6$ years; baseline off-state Unified Parkinson's Disease Rating Scale [UPDRS] motor rating 24.6 \pm 6.8) at The Feinstein Institute for Medical Research. The diagnosis of PD was made according to the United Kingdom (UK) Brain Bank Criteria for idiopathic PD (53). All subjects exhibited greater than $20 \%$ improvement in UPDRS motor ratings following a single oral dose of levodopa/carbidopa administered at the time of enrollment. See Supplemental Methods for detailed information about these and the second set of 106 subjects from Cologne.

PET. Infusion subjects fasted overnight and were off antiparkinsonian medications for at least 12 hours before undergoing dual-tracer imaging with $\left[{ }^{15} \mathrm{O}\right]$ water $\left(\mathrm{H}_{2}{ }^{15} \mathrm{O}\right)$ and FDG PET to map CBF and CMR at baseline $(\mathrm{OFF})$ and during levodopa treatment $(\mathrm{ON})$ in separate randomly ordered sessions over a 2-day period (17). Details of the procedure, including the levodopa infusion, scanning protocol, and image processing are provided in Supplemental Methods.

Detection of regions with significant dissociation of vasomotor and metabolic responses to levodopa. Unbiased whole brain voxel-wise searches were conducted to identify regions with significant dissociation of CBF and CMR responses to levodopa. To this end, dual-tracer scan data from the LID and NLID groups were separately interrogated for clusters with significant function $(\mathrm{CBF} / \mathrm{CMR}) \times$ treatment $(\mathrm{OFF} / \mathrm{ON})$ interaction effects using SPM5 software (http://www.fil.ion.ucl.ac.uk/spm/software/spm5/). In this analysis, CBF and CMR scans obtained in both treatment conditions were globally normalized at a relative signal threshold of 0.8 . Searches for significant interaction effects were defined by the contrasts $\left[\mathrm{CBF}_{\mathrm{ON}}-\mathrm{CBF}_{\mathrm{OFF}}\right]>\left[\mathrm{CMR}_{\mathrm{ON}}-\mathrm{CMR}_{\mathrm{OFF}}\right]$ and $\left[\mathrm{CBF}_{\mathrm{ON}}-\mathrm{CBF}_{\mathrm{OFF}}\right]<\left[\mathrm{CMR}_{\mathrm{ON}}-\mathrm{CMR}_{\mathrm{OFF}}\right]$. The resulting maps were thresholded at $P<0.001$ (voxel-level, uncorrected); clusters were considered to be significant if they survived family-wise error (FWE) correction for multiple comparisons at $P<0.05$. 
For each region with significant levodopa-mediated $\mathrm{CBF} / \mathrm{CMR}$ dissociation, a spherical VOI (radius $=3$ $\mathrm{mm}$ ) was centered at the peak voxel of the corresponding cluster. For each VOI, we computed globally normalized CBF and CMR values for each PD subject in the LID, NLID, and TRT groups in both treatment conditions (on- and off-levodopa for the LID and NLID groups; test and retest for the TRT group). For regions found to be bilaterally significant, VOI values were averaged for the left and right hemispheres. In the case of unilaterally significant regions, values were averaged for VOIs obtained at peak voxel and at the mirror coordinates on the opposite hemisphere. The changes observed in the regions with significant levodopa-mediated dissociation were compared with corresponding effects measured in a neutral reference region. We chose the occipital lobe as the reference region for these analyses because of its low DDC expression (25). Occipital reference values were computed in the on- and off-state CBF and CMR scans from each subject using a region of interest (ROI) defined in common space according to the Automated Anatomical Labeling (AAL) atlas (54).

We computed DI values, which quantify the degree of local dissociation of vasomotor and metabolic levodopa responses on an individual-subject basis, for each of the significant regions identified by voxel-wise analysis (see above) and for the occipital reference region. DI was defined as the difference between $\triangle \mathrm{CBF}_{\mathrm{ON}-\mathrm{OFF}}$ and $\triangle \mathrm{CMR}_{\mathrm{ON}-\mathrm{OFF}}$ in a given brain region for a particular individual (17). Differences in DI values across the LID, NLID, and TRT groups were assessed using 1-way ANOVA followed by post hoc Bonferroni tests. $\chi^{2}$ tests were also used to examine whether the proportion of positive DI values in the NLID or LID group differed from that of the TRT group. In addition, we separately evaluated group $\times$ condition interaction effects in the corresponding CBF and CMR data for each region, using $2 \times 2$ RMANOVA followed by post hoc Bonferroni tests.

Last, we determined whether levodopa infusion altered baseline relationships between CBF and CMR in significant dissociation regions. This was done for each region using regression analysis in which local $\mathrm{CBF}$ and CMR values were correlated in the on- and off-conditions separately. This analysis was conducted on the LID and NLID data separately and in combination. Uncoupling was defined as treatment-mediated loss of the significant baseline correlation between $\mathrm{CBF}$ and $\mathrm{CMR}$ that is present in that brain region in $\mathrm{PD}$ subjects. Specifically, we used the Chow test (55) to evaluate the null hypothesis that no change in regression coefficients occurred with treatment. In the current context, uncoupling may occur in brain regions that exhibit high degrees of levodopa-mediated dissociation.

Validation of regional dissociation effects. We searched for areas of significant levodopa-mediated CBF/ CMR dissociation on an individual group basis. To avoid potential bias from concurrent involuntary movements (dyskinesias) in the on-state scans of members of the LID group, we first sought out dissociated regions in a whole brain voxel-wise analysis of the NLID scans. The resulting coordinates were applied post hoc to individual CBF and CMR scans from the LID and TRT subjects, and corresponding globally normalized VOI values were computed for each subject and treatment condition.

For validation, we performed a complementary voxel-wise search to identify areas with significant CBF/CMR dissociation in the LID data. By analogy to the post hoc analysis of the NLID-derived dissociation regions, VOIs representing significant dissociation regions identified in the LID analysis were applied post hoc to individual CBF and CMR scan data from the NLID and TRT groups. We hypothesized that voxel-wise searches for areas with significant dissociation areas would yield analogous results (i.e., clusters with similar location and effect size) whether derived from LID or NLID data. That is, if truly unbiased by the presence of dyskinesias, the location and relative magnitude of the observed dissociation effects would not be altered if the significant regions are independently identified in scans from LID rather than NLID subjects. To test this hypothesis, we computed individual subject values for DI, $\triangle \mathrm{CBF} \mathrm{ON}$-OFF, $_{\text {, and }}$ $\triangle \mathrm{CMR}_{\mathrm{ON}-\mathrm{OFF}}$, and for on- and off-state $\mathrm{CBF}$ and CMR for corresponding NLID- and LID-based VOIs, and compared them by computing Pearson product-moment correlation coefficients.

LID versus NLID: baseline differences. In addition to brain regions that differentiate LID from NLID subjects based upon local levodopa-mediated dissociation effects, we interrogated the scan data for areas in which the 2 groups were distinguished by baseline (off-state) indices of local cerebral function, i.e., by CBF and/or CMR values measured in the absence of medication. To identify such regions, we conducted whole brain voxel-wise searches for clusters with significant $\operatorname{LID}_{\mathrm{OFF}}>\mathrm{NLID}_{\mathrm{OFF}}$ or $\mathrm{LID}_{\mathrm{OFF}}<\mathrm{NLID}_{\mathrm{OFF}}$ differences. As above, the resulting maps were thresholded at $P<0.001$ (voxel-level, uncorrected). Given the exploratory nature of these analyses, we reported regional findings at this threshold, with a cluster size cutoff of $k$ $\geq 100$ voxels. VOI analysis was conducted as above to compute on- and off-state CBF and CMR values for each subject in the significant brain regions. 
Relationship between regional changes and local dopaminergic input. Because of radiation dosimetry constraints, it was not possible to perform additional imaging with FDOPA PET in the current patient sample. It was, however, possible to define VOIs corresponding to the areas identified as having significant levodopa-mediated CBF/CMR dissociation or significant baseline differences in these subjects and transfer the regions to coregistered FDOPA PET slices from a separate, although clinically and demographically very similar, group of patients from Cologne (see Supplemental Methods).

Clinical correlates of levodopa-mediated dissociation effects. We utilized logistic regression analysis to determine the accuracy with which putamen DI, as well as the local vasomotor and metabolic levodopa response, discriminated LID from NLID subjects - either as individual measures, or in combination with baseline SMC values. Model selection was based on relative goodness of fit as determined by the Akaike information criterion (AIC) (56). For each model, we computed the max-rescaled $r^{2}$ value, the $P$ value of the likelihood ratio test, and the AUC that resulted from the corresponding ROC analysis.

We also correlated vasomotor and metabolic levodopa responses ( $\triangle \mathrm{CBF}$ and $\triangle \mathrm{CMR}$, respectively) in the significant regions with independent disease descriptors: symptom duration (as reported by the patient and/or the clinical record) and $\operatorname{LDD}(\mathrm{w})(\mathrm{mg} / \mathrm{kg} / \mathrm{d})$ at the time of the study. The latter measure exhibited a significant correlation with cumulative levodopa exposure $(r=0.76, P<0.007)$ estimated in 11 of the PD subjects ( 3 NLID; 8 LID) for whom histories of medication dose were available through detailed chart review. As an objective image-based descriptor of disease progression for each subject, expression values (subject scores) for the PD-related metabolic covariance pattern (PDRP) $(12,13)$ were quantified in the off-state CMR scans that were acquired as part of the current study. These values were computed using an automated network algorithm described in detail elsewhere $(11,57)$. Relationships between these disease descriptors and local vasomotor $(\triangle \mathrm{CBF})$ and metabolic $(\triangle \mathrm{CMR})$ levodopa responses recorded in relevant brain regions were evaluated using multiple regression analysis. Regression models were constructed based upon single and multiple predictor variables; $\triangle \mathrm{CBF}$ and $\triangle \mathrm{CMR}$ responses were evaluated using separate predictive models. The effect of each predictor on the regional drug response was assessed graphically using leverage plots (partial correlations) in which the residuals for the dependent variable were plotted against those for each of the predictors after removing the other effects in the model.

Statistics. Differences in demographic features and clinical measures between PD groups were evaluated using Student's $t$ tests or $\chi^{2}$ tests, e.g., to compare gender distributions across samples. For each VOI with significant levodopa-mediated CBF/CMR dissociation effects (see above), differences in regional DI across the LID, NLID, and TRT groups were evaluated using 1-way ANOVA with post hoc Bonferroni tests. Group $\times$ condition interaction effects in the $\mathrm{CBF}$ and $\mathrm{CMR}$ data were assessed for each VOI using $2 \times 2$ RMANOVA with post hoc Bonferroni tests. The relationship between CBF and CMR in regions with significant dissociation effects was assessed by computing Pearson product-moment correlation coefficients for the off- and onlevodopa conditions. To assess levodopa-mediated uncoupling of CBF and CMR in each of these regions, we evaluated changes in regression coefficients across conditions using Chow tests (55).

To validate the observed dissociation effects, DI values measured in the VOIs identified in the NLID data were correlated with corresponding values for VOIs identified by separate analysis of the LID data. For regions defined by significant baseline CBF differences between LID and NLID subjects, group $\times$ condition interaction effects were examined using $2 \times 2$ RMANOVA with post hoc Bonferroni tests. On- and off- state $\mathrm{CBF}$ and CMR values in significant brain regions, and the corresponding vasomotor $(\triangle \mathrm{CBF})$ and metabolic $(\triangle \mathrm{CMR})$ levodopa responses, were correlated with demographic and clinical disease descriptors by computing Pearson product-moment correlation coefficients for the LID and NLID groups. Levodopa-mediated changes in CBF and CMR in each region were assessed for individual subjects separately in the LID and NLID groups with nonparametric binomial tests. CBF and CMR values measured off- and on-levodopa for each region were compared with corresponding healthy control values using 2-tailed Student's $t$ tests. An analogous approach was used on the Cologne data (see Supplemental Methods) to compare baseline dissociation VOI measurements of FDOPA uptake and CMR in subjects with mild and advanced PD, and to compare patient with corresponding healthy control values.

Logistic regression analysis with hierarchical model selection (56) was used to determine the accuracy of discrimination between LID and NLID that was achieved with $\triangle \mathrm{CBF}$ or $\triangle \mathrm{CMR}$, or with local DI values. We also determined whether discrimination accuracy could be improved by adding baseline SMC values into the logistic model. Last, we used multiple regression to explore relationships between 
putamen vasomotor and metabolic levodopa responses on the one hand, and independent measures of disease [symptom duration, $\mathrm{LDD}(\mathrm{w})$, and PDRP expression] on the other.

All statistical analyses were performed in SAS 9.3, and the results were considered significant at $P<0.05$.

Study approval. Ethical permission for the studies was obtained from the Institutional Review Board of Northwell Health and the ethics committee of the Medical Faculty of the University of Cologne. For subjects scanned at The Feinstein Institute for Medical Research, written consent was obtained from each subject after detailed explanation of the procedures. Information on informed consent for subjects scanned at Cologne University Hospital has been reported elsewhere (22).

\section{Author contributions}

VAJ acquired and analyzed the data, and prepared the manuscript; CCT oversaw the statistical analysis of the data; FH and CD acquired and analyzed the data; YYC assisted with the data analysis and helped prepare the manuscript; YM performed large-scale voxel searches of the Cologne data; VD conducted and supervised the imaging experiments; and DE designed the research study, and supervised the conduct of the experiments, the analysis and interpretation of the data, and the writing of the manuscript.

\section{Acknowledgments}

This work was supported by the National Institute of Neurological Disorders and Stroke Morris K. Udall Center of Excellence for Parkinson's Disease Research at The Feinstein Institute for Medical Research (P50 NS071675 to DE). The content is solely the responsibility of the authors and does not necessarily represent the official views of the National Institute of Neurological Disorders and Stroke or the National Institutes of Health. The sponsor did not play a role in study design, collection, analysis, and interpretation of data, writing of the report, or in the decision to submit the paper for publication. VAJ is the recipient of a fellowship award from the Fonds de Recherche en Santé du Québec. FH was supported by Deutsche Forschungsgemeinschaft. The authors thank Toni Fitzpatrick and Michael Small for their assistance in preparing the manuscript for publication, and V.L. Brandt for invaluable comments and editorial advice.

Address correspondence to: David Eidelberg, 350 Community Drive, Manhasset, New York 11030, USA. Phone: 516.562.2498; E-mail: deidelbe@northwell.edu.

FH's present address is: Department of Neurology, RWTH Aachen University, Aachen, Germany.

1. Connolly BS, Lang AE. Pharmacological treatment of Parkinson disease: a review. JAMA. 2014;311(16):1670-1683

2. Fahn S, Poewe W. Levodopa: 50 years of a revolutionary drug for Parkinson disease. Mov Disord. 2015;30(1):1-3.

3. Van Gerpen JA, Kumar N, Bower JH, Weigand S, Ahlskog JE. Levodopa-associated dyskinesia risk among Parkinson disease patients in Olmsted County, Minnesota, 1976-1990. Arch Neurol. 2006;63(2):205-209.

4. Cenci MA. Presynaptic mechanisms of 1-dopa-induced dyskinesia: the findings, the debate, and the therapeutic implications. Front Neurol. 2014;5:242.

5. Stoessl AJ. Central pharmacokinetics of levodopa: lessons from imaging studies. Mov Disord. 2015;30(1):73-79.

6. Politis M, et al. Serotonergic mechanisms responsible for levodopa-induced dyskinesias in Parkinson's disease patients. J Clin Invest. 2014;124(3):1340-1349.

7. Baron JC, et al. Local interrelationships of cerebral oxygen consumption and glucose utilization in normal subjects and in ischemic stroke patients: a positron tomography study. J Cereb Blood Flow Metab. 1984;4(2):140-149.

8. Herscovitch P. Can [15O]water be used to evaluate drugs? J Clin Pharmacol. 2001;Suppl:11S-20S.

9. Clarke DE, and Sokoloff L. Circulation and energy metabolism of the brain. In Siegel GJ, ed. Basic Neurochemistry. Philadelphia, Pennsylvania, USA: Lippincott; 1999. http://www.ncbi.nlm.nih.gov/books/NBK20413. Accessed August 19, 2016.

10. Dhawan V, Tang CC, Ma Y, Spetsieris P, Eidelberg D. Abnormal network topographies and changes in global activity: absence of a causal relationship. Neuroimage. 2012;63(4):1827-1832.

11. Spetsieris PG, Eidelberg D. Scaled subprofile modeling of resting state imaging data in Parkinson's disease: methodological issues. Neuroimage. 2011;54(4):2899-2914.

12. Eidelberg D. Metabolic brain networks in neurodegenerative disorders: a functional imaging approach. Trends Neurosci. 2009;32(10):548-557.

13. Niethammer M, Eidelberg D. Metabolic brain networks in translational neurology: concepts and applications. Ann Neurol. 2012;72(5):635-647.

14. Tang CC, et al. Differential diagnosis of parkinsonism: a metabolic imaging study using pattern analysis. Lancet Neurol. 2010;9(2):149-158.

15. Ko JH, et al. Network modulation following sham surgery in Parkinson's disease. J Clin Invest. 2014;124(8):3656-3666.

16. Asanuma K, et al. Network modulation in the treatment of Parkinson's disease. Brain. 2006;129(Pt 10):2667-2678. 
17. Hirano S, et al. Dissociation of metabolic and neurovascular responses to levodopa in the treatment of Parkinson's disease. J Neurosci. 2008;28(16):4201-4209.

18. Afonso-Oramas D, et al. Striatal vessels receive phosphorylated tyrosine hydroxylase-rich innervation from midbrain dopaminergic neurons. Front Neuroanat. 2014;8:84.

19. Edvinsson L, Krause D. Catecholamines. In Edvinsson L, Krause D, eds. Cerebral Blood Flow and Metabolism. Philadelphia, Pennsylvania, USA: Lippincott Wiliams \& Wilkins; 2002: 191-211.

20. Ohlin KE, Sebastianutto I, Adkins CE, Lundblad C, Lockman PR, Cenci MA. Impact of L-DOPA treatment on regional cerebral blood flow and metabolism in the basal ganglia in a rat model of Parkinson's disease. Neuroimage. 2012;61(1):228-239.

21. Tang CC, Poston KL, Dhawan V, Eidelberg D. Abnormalities in metabolic network activity precede the onset of motor symptoms in Parkinson's disease. J Neurosci. 2010;30(3):1049-1056.

22. Holtbernd F, et al. Dopaminergic correlates of metabolic network activity in Parkinson's disease. Hum Brain Mapp. 2015;36(9):3575-3585

23. Moore RY, Whone AL, Brooks DJ. Extrastriatal monoamine neuron function in Parkinson's disease: an 18F-dopa PET study. Neurobiol Dis. 2008;29(3):381-390.

24. de la Fuente-Fernández R, et al. Levodopa-induced changes in synaptic dopamine levels increase with progression of Parkinson's disease: implications for dyskinesias. Brain. 2004;127(Pt 12):2747-2754.

25. Ishikawa T, et al. Clinical significance of striatal DOPA decarboxylase activity in Parkinson's disease. J Nucl Med. 1996;37(2):216-222.

26. Pavese N, Simpson BS, Metta V, Ramlackhansingh A, Chaudhuri KR, Brooks DJ. $\left[{ }^{18} \mathrm{~F}\right]$ FDOPA uptake in the raphe nuclei complex reflects serotonin transporter availability. A combined $\left[{ }^{18} \mathrm{~F}\right] \mathrm{FDOPA}$ and $\left[{ }^{11} \mathrm{C}\right] \mathrm{DASB}$ PET study in Parkinson's disease Neuroimage. 2012;59(2):1080-1084.

27. Feigin A, et al. Metabolic correlates of levodopa response in Parkinson's disease. Neurology. 2001;57(11):2083-2088.

28. Luquin MR, Scipioni O, Vaamonde J, Gershanik O, Obeso JA. Levodopa-induced dyskinesias in Parkinson's disease: clinical and pharmacological classification. Mov Disord. 1992;7(2):117-124.

29. Goetz CG, et al. Placebo response in Parkinson's disease: comparisons among 11 trials covering medical and surgical interventions. Mov Disord. 2008;23(5):690-699.

30. Sokoloff L. Energetics of functional activation in neural tissues. Neurochem Res. 1999;24(2):321-329.

31. Lin TP, et al. Metabolic correlates of subthalamic nucleus activity in Parkinson's disease. Brain. 2008;131(Pt 5):1373-1380.

32. Lee JY, Seo S, Lee JS, Kim HJ, Kim YK, Jeon BS. Putaminal serotonergic innervation: monitoring dyskinesia risk in Parkinson disease. Neurology. 2015;85(10):853-860.

33. Halje P, Tamtè M, Richter U, Mohammed M, Cenci MA, Petersson P. Levodopa-induced dyskinesia is strongly associated with resonant cortical oscillations. J Neurosci. 2012;32(47):16541-16551.

34. Cenci MA, Konradi C. Maladaptive striatal plasticity in L-DOPA-induced dyskinesia. Prog Brain Res. 2010;183:209-233.

35. Westin JE, et al. Endothelial proliferation and increased blood-brain barrier permeability in the basal ganglia in a rat model of 3,4-dihydroxyphenyl-L-alanine-induced dyskinesia. J Neurosci. 2006;26(37):9448-9461.

36. Lindgren HS, Ohlin KE, Cenci MA. Differential involvement of D1 and D2 dopamine receptors in L-DOPA-induced angiogenic activity in a rat model of Parkinson's disease. Neuropsychopharmacology. 2009;34(12):2477-2488.

37. Ohlin KE, et al. Vascular endothelial growth factor is upregulated by L-dopa in the parkinsonian brain: implications for the development of dyskinesia. Brain. 2011;134(Pt 8):2339-2357.

38. Adair TH, Montani JP. Angiogenesis. San Rafael, California, USA: Morgan \& Claypool Life Sciences; 2010. http://www.ncbi. nlm.nih.gov/books/NBK53242. Accessed August 19, 2016.

39. Vogel J, Gehrig M, Kuschinsky W, Marti HH. Massive inborn angiogenesis in the brain scarcely raises cerebral blood flow. J Cereb Blood Flow Metab. 2004;24(8):849-859.

40. Filosa JA. Vascular tone and neurovascular coupling: considerations toward an improved in vitro model. Front Neuroenergetics. $2010 ; 2$.

41. Uc EY, Dienel GA, Cruz NF, Harik SI. beta-Adrenergics enhance brain extraction of levodopa. Mov Disord. 2002;17(1):54-59.

42. Gray MT, Woulfe JM. Striatal blood-brain barrier permeability in Parkinson's disease. J Cereb Blood Flow Metab. 2015;35(5):747750.

43. Cilia R, et al. The modern pre-levodopa era of Parkinson's disease: insights into motor complications from sub-Saharan Africa. Brain. 2014;137(Pt 10):2731-2742.

44. Holtbernd F, et al. Abnormal metabolic network activity in REM sleep behavior disorder. Neurology. 2014;82(7):620-627.

45. Wu P, et al. Consistent abnormalities in metabolic network activity in idiopathic rapid eye movement sleep behaviour disorder. Brain. 2014;137(Pt 12):3122-3128.

46. Spetsieris PG, et al. Metabolic resting-state brain networks in health and disease. Proc Natl Acad Sci USA. 2015;112(8):2563-2568.

47. Lewitt PA, et al. Randomized clinical trial of fipamezole for dyskinesia in Parkinson disease (FJORD study). Neurology. 2012;79(2):163-169.

48. Perez XA. Preclinical evidence for a role of the nicotinic cholinergic system in Parkinson's disease. Neuropsychol Rev. 2015;25(4):371-383.

49. Trost M, et al. Network modulation by the subthalamic nucleus in the treatment of Parkinson's disease. Neuroimage. 2006;31(1):301-307.

50. Wang J, Ma Y, Huang Z, Sun B, Guan Y, Zuo C. Modulation of metabolic brain function by bilateral subthalamic nucleus stimulation in the treatment of Parkinson's disease. J Neurol. 2010;257(1):72-78.

51. Ko JH, et al. Parkinson's disease: increased motor network activity in the absence of movement. J Neurosci. 2013;33(10):4540-4549.

52. Mure H, et al. Improved sequence learning with subthalamic nucleus deep brain stimulation: evidence for treatment-specific network modulation. J Neurosci. 2012;32(8):2804-2813

53. Hughes AJ, Daniel SE, Kilford L, Lees AJ. Accuracy of clinical diagnosis of idiopathic Parkinson's disease: a clinico-pathological study of 100 cases. J Neurol Neurosurg Psychiatr. 1992;55(3):181-184. 
54. Tzourio-Mazoyer N, et al. Automated anatomical labeling of activations in SPM using a macroscopic anatomical parcellation of the MNI MRI single-subject brain. Neuroimage. 2002;15(1):273-289.

55. Chow GC. Tests of equality between sets of coefficients in two linear regressions. Econometrica. 1960;28(3):591-605.

56. Burnham KP, and Anderson DR. Model Selection and Multimodel Inference. New York, New York, USA: Springer Verlag; 2002.

57. Spetsieris $\mathrm{P}$, et al. Identification of disease-related spatial covariance patterns using neuroimaging data. J Vis Exp. 2013;(76). 